\title{
Small mesoscopic particles in dilute and semidilute solutions of nonadsorbing polymers
}

\author{
E. Eisenriegler \\ Institut für Festkörperforschung, Forschungszentrum Jülich, D-52425 Jülich, Germany
}

(Received 3 May 2000; accepted 26 June 2000)

\begin{abstract}
Polymer-induced interactions between two small mesoscopic particles of radius $R$ and between a particle and a wall are investigated for dilute or semidilute embedding solutions of long flexible nonadsorbing polymer chains with radius of gyration $\mathcal{R}_{g}$. Asymptotically exact predictions are obtained using a "small radius expansion," to express the interactions in terms of properties of the polymer solution without particles. The nonmonotonic dependence of the second virial coefficient $B_{2}^{C C}$ of a dilute suspension of particles on the interchain overlap is discussed in detail. The magnitude of the minimum of $B_{2}^{\mathrm{CC}} / R^{3}$ increases as a power law in $\mathcal{R}_{g} / R$. The exponent and amplitude are quite different from the earlier prediction of an integral-equation approach. For dilute polymers in two dimensions outside two circular disks in contact, exact results are given for the monomer-density depletion profile, the pressure variation along the perimeter of, and the polymer-induced force between the two disks. (C) 2000 American Institute of Physics.
\end{abstract}

[S0021-9606(00)50536-4]

\section{INTRODUCTION}

Colloidal particles embedded in a solution of nonadsorbing polymer chains experience a depletion interaction. For entropic reasons the chains avoid the space between two close particles, leading to an unbalanced pressure from outside which pushes the two particles towards each other. This mechanism is believed to be important for a variety of interesting colloids such as casein micelles, ${ }^{1}$ red blood cells, ${ }^{2}$ and globular proteins. ${ }^{3}$

Consider spherical particles of mesoscopic size dissolved in extreme dilution in a monodisperse solution of long flexible nonadsorbing polymer chains in a good solvent. On mesoscopic length scales and disregarding long range forces ${ }^{4}$ the system is equivalent to a model containing only particles and chains in which the interactions both between a chainmonomer and a particle and between two chain-monomers (belonging to the same or to different chains) are of pure excluded volume type. ${ }^{5,6}$ The polymer-induced depletion interaction between the particles has a rich structure which depends on the ratio of the particle and chain sizes ${ }^{7}$ and on the degree of overlap between the chains, i.e., on whether the polymer solution is dilute or semidilute. ${ }^{8}$

For large particle size the depletion interaction closely resembles that for planar walls. ${ }^{9,10}$ Here the focus is on small spherical particles whose radius $R$, though large on a microscopic scale, is much smaller than the radius of gyration $\mathcal{R}_{g}$ of an isolated chain in the dilute solution or the Edwards screening length (or "blob" size) " $^{5,6} \xi$ in the semidilute solution. At a first glance the small particle case looks complicated, since it is dominated by configurations in which a chain coils around the particle and approximations treating the chains as nondeformable ${ }^{9}$ fail completely. Probably this is one of the reasons why previous theoretical investigations concentrated more on the case of large particles. However, for understanding, e.g., the role of polymers such as polyethylene glycol in the precipitation and crystallization of proteins, ${ }^{11}$ the small particle case is also of interest. ${ }^{3}$

Asymptotically exact results can be given for particle radius $R$ much smaller than the other relevant mesoscopic lengths, since the particle can be viewed as a weakly repulsive point-perturbation for chain monomers. With the center of the colloidal particle at $\mathbf{r}_{C}$ this leads ${ }^{12,13}$ to a reduction factor

$$
W_{C}=1-A_{g} R^{d-1 / \nu} \mathcal{R}_{g}^{1 / \nu} \theta\left(\mathbf{r}_{C}\right)
$$

in the Boltzmann weight for chain configurations. Here $A_{g}$ is a dimensionless amplitude discussed below, $d$ is the spatial dimension, ${ }^{14} \nu$ is the Flory exponent, ${ }^{5} \mathcal{R}_{g}$ is the radius of gyration of a single chain in dilute solution as introduced above, and

$$
\theta(\mathbf{r})=\sum_{P} \frac{1}{N} \sum_{j=1}^{N} \delta\left(\mathbf{r}-\mathbf{r}_{P, j}\right)
$$

is the configuration-dependent number density of chains. The sum $\Sigma_{P}$ runs over all polymer chains $P$ in the solution, $\mathbf{r}_{P, j}$ is the position of monomer $j$ in chain $P$, and $N$ is the number of monomers per chain. Integrating $\theta$ over a volume element $d \mathbf{r}$, the contribution of each chain $P$ equals the fraction of its monomers which, in a given configuration of the chains, are found inside $d \mathbf{r}$. Unlike $N$ this fraction is independent of the precise definition of the monomer. The configurational average of $\theta$ in a solution without particles equals the usual number density $n$ of chains in the solution. The dimensionless amplitude $A_{g}$ in Eq. (1.1) is universal, i.e., independent of details of the long flexible polymer chains, in the same sense as the Flory exponent $\nu$. For chains in good solvent in $d$ $=3$ spatial dimensions, ${ }^{15}$

$A_{g} \approx 18.4 ; \quad d=3, \quad$ good solvent. 
Equation (1.1) describes the effective repulsion of the small particle quantitatively. For example, the free energy $F_{1}$ it costs to insert one particle in the bulk solution takes the form $^{12,13}$

$$
\frac{F_{1}}{k_{B} T}=A_{g} R^{d-1 / \nu} n \mathcal{R}_{g}^{1 / \nu},
$$

where $n$ is the number density of chains in the pure bulk solution introduced above. This result, which follows from Eqs. (1.1) and (1.2), applies for arbitrary values of the overlap $^{6}$

$$
s=n \mathcal{R}_{g}^{d}
$$

between chains. In the semidilute limit in which $s \rightarrow \infty$ with the monomer density $\propto_{n} \mathcal{R}_{g}^{1 / \nu}$ kept fixed at some small value, the screening length $\xi$ remains finite, and $A_{g}$ provides the numerical value of the amplitude $a$ in the result of de Gennes ${ }^{16} F_{1} / k_{B} T=a(R / \xi)^{d-1 / \nu}$, since $a / A_{g}$ equals the well known amplitude $\mathcal{R}_{g}^{1 / \nu} n \xi^{d-1 / \nu}$ of a semidilute bulk solution. ${ }^{5,6}$ For the case of a good solvent in three dimensions, the exponent $d-1 / \nu$ in Eq. (1.4) equals 1.3 , and $F_{1}$ decreases more rapidly with decreasing $R$ than the prediction $F_{1} \propto R$ of mean field ${ }^{17}$ or integral equation ${ }^{8}$ approaches.

In this paper we derive asymptotically exact results for other interesting properties of small particles dissolved in a polymer solution with arbitrary overlap $s$ between the chains. In Sec. II we consider the polymer-induced free energy of interaction (or potential of mean force) $\delta F_{2}$ between two particles. For two spheres in contact we discuss in Sec. III the depletion profile of the monomer density and the local pressure which varies along the surface of the spheres. Section IV presents results for the second virial coefficient $B_{2}^{\mathrm{CC}}$ of a dilute suspension of colloidal particles. The second virial coefficient, which can be measured by light scattering, is of particular interest, since in protein suspensions its value appears to be correlated with the success of protein crystallization. ${ }^{18}$ We make contact with the results of Chatterjee and Schweizer, ${ }^{8}$ based on an integral equation approach for arbitrary overlap and with the results of Sear ${ }^{19}$ for the semidilute limit. Our quantitative results confirm the qualitative behavior of the dependence of $B_{2}^{\mathrm{CC}}$ on polymer concentration with a minimum at $n(4 \pi / 3) \mathcal{R}_{g}^{3} \approx 1$, as pointed out in Ref. 8. However, the dependence of the minimum value of $B_{2}^{\mathrm{CC}}$ on $\mathcal{R}_{g} / R$ differs significantly from Ref. 8, as discussed in Secs. IV and VI.

In Sec. $\mathrm{V}$ the overlap-dependence of the polymerinduced interaction of a particle with a wall is considered. Section VI contains a summary and concluding remarks.

\section{POTENTIAL OF MEAN FORCE BETWEEN TWO PARTICLES}

If two particles $A$ and $B$ are immersed in the polymer solution with their centers at $\mathbf{r}_{A}$ and $\mathbf{r}_{B}$, the Boltzmann weight of each polymer configuration is reduced by $W_{A} W_{B}$. This factor is the product of reduction factors for single particles, each of which has the form (1.1) if the particles are small, i.e., if $R \ll r_{A B}, \mathcal{R}_{g}, \xi$ with the center-to-center distance $r_{A B}=\left|\mathbf{r}_{A}-\mathbf{r}_{B}\right|$. The free energy $F_{2}$ of immersion of the two particles is the difference in polymer free energy in the presence and absence of the particles, i.e., $F_{2}$ $=-k_{B} T \ln \left\langle W_{A} W_{B}\right\rangle$, where the angular brackets denote an average over all chain configurations in the bulk solution without particles. The free energy of interaction or potential of mean force between the two particles

$$
\delta F_{2}=-k_{B} T \ln \frac{\left\langle W_{A} W_{B}\right\rangle}{\left\langle W_{A}\right\rangle\left\langle W_{B}\right\rangle}
$$

is the difference between $F_{2}$ and the sum $2 F_{1}$ of the free energies $-k_{B} T \ln \left\langle W_{A}\right\rangle$ and $-k_{B} T \ln \left\langle W_{B}\right\rangle$ for immersing $A$ without $B$ and $B$ without $A$. Clearly $\delta F_{2}$ depends on $r_{A B}$ and tends to zero for $r_{A B} \rightarrow \infty$. On expanding $\delta F_{2}$ in terms of $W_{A}-1$ and $W_{B}-1$ for small particles, the self-terms of first and second order drop out, and

$$
\begin{aligned}
\frac{\delta F_{2}}{k_{B} T} & =-\left[\left\langle\left(W_{A}-1\right)\left(W_{B}-1\right)\right\rangle-\left\langle W_{A}-1\right\rangle\left\langle W_{B}-1\right\rangle\right] \\
& =-\left(\frac{F_{1}}{k_{B} T n}\right)^{2} K\left(r_{A B}\right), \quad R \ll r_{A B}, \mathcal{R}_{g}, \xi,
\end{aligned}
$$

where

$$
K\left(r_{A B}\right)=\left\langle\theta\left(\mathbf{r}_{A}\right) \theta\left(\mathbf{r}_{B}\right)\right\rangle-n^{2}
$$

is the normalized polymer density correlation function in the solution without particles. Here $\theta$ is defined in Eq. (1.2), and Eqs. (1.1) and (1.4) have been used in the second step of Eq. (2.2). Equations (2.2) and (2.3) generalize Eq. (8) of Sear, ${ }^{19}$ valid for the semidilute limit, to arbitrary overlap and provide the missing prefactor in this relation.

If the distance $r_{A B}$ between the two particles is much smaller than $\mathcal{R}_{g}$ and $\xi$, the quantity $K$ in Eq. (2.2) is given by the density correlation deep inside a single polymer coil, ${ }^{6}$ and $^{13}$

$$
K\left(r_{A B}\right) \rightarrow n \frac{\sigma_{g}}{\mathcal{R}_{g}^{1 / \nu} r_{A B}^{d-1 / \nu}} ; \quad r_{A B} \ll \mathcal{R}_{g}, \xi
$$

increases linearly with the chain density $n$ throughout the whole dilute to semidilute concentration range. Here $\sigma_{g}$ is a known universal amplitude with the value ${ }^{15}$

$$
\sigma_{g} \approx 0.069 ; \quad d=3, \quad \text { good solvent. }
$$

In the limit of a dilute polymer solution $K\left(r_{A B}\right)$ reduces to $n C_{2}\left(r_{A B}\right) / \mathcal{R}_{x}^{2 / \nu}$, with $C_{2}$ the $n$-independent monomer density pair correlation function introduced in Eq. (1.17) of Ref. 13.

For distances $r_{A, B}$ between the two particles in the regime (2.4), the ratio $F_{2}\left(r_{A, B}\right) / F_{2}(\infty)$ deviates from 1 by an amount proportional to $-\left(R / r_{A, B}\right)^{d-1 / \nu}$, which is independent of $\mathcal{R}_{g}$ and $\xi$ (and thus independent of the overlap) and suggests that the range of the interaction between two small spheres is of the order of their radius $R$. However, this power law is not integrable. Integrals such as the second virial coefficient given in Eq. (4.1) below are dominated by much larger distances $r_{A, B}$ of the order of $\mathcal{R}_{g}$ or $\xi$ and have a nontrivial overlap-dependence.

Strictly speaking the expression in Eq. (2.2) only applies to distances $r_{A B}$ between the two particles which are much 
larger than their radius $R$ and should be compared with the potential of mean force $\left(\delta F_{2} / k_{B} T\right)_{r_{A B}=2 R}$ for the smallest possible distance $r_{A B}=2 R$, i.e., for two spheres in contact. This has the form

$$
\left(\frac{\delta F_{2}}{k_{B} T}\right)_{r_{A B}=2 R}=-(2-m) \frac{F_{1}}{k_{B} T},
$$

where $m F_{1}$ is the free energy of immersion of a pair of spheres in contact. ${ }^{20}$ The value of $m$ for polymers in good solvent equals $(\pi / 2)^{2 / 3}$ in $d=2$ while near the upper critical dimension $^{5}$ in $d=4-\epsilon$ it follows ${ }^{20}$ from the $\epsilon$-expansion of the energy density profile in a critical Ginzburg-Landau model between parallel plates ${ }^{21}$ as

$$
\frac{m}{m_{\mathrm{id}}}=1+\frac{\epsilon}{4}\left(\ln \frac{\pi}{2}-\frac{1}{4}\right)=1+0.0504 \epsilon .
$$

Here $m_{\mathrm{id}}=2\left(1-2^{3-d}\right) \zeta(d-2)$ is the value of $m$ for ideal polymers with $\zeta$ the Riemann zeta function. Enhancement of $m$ due to monomer-monomer repulsion as implied by Eq. (2.7) is also found for the case $d \rightarrow 2 \quad(\epsilon \rightarrow 2)$ in which $\mathrm{m} / \mathrm{m}_{\mathrm{id}} \rightarrow m=1.35$.

Extrapolating expressions (2.2) and (2.4) to $r_{A B}=2 R$ also leads to the form (2.6), with $2-m$ replaced by $A_{g} \sigma_{g} / 2^{d-1 / \nu}$. Comparison of the two amplitudes shows that for three dimensions and good solvent the extrapolation approximates the amplitude $2-m \approx 0.5$ in Eq. (2.6) rather well.

\section{POLYMER DENSITY, PRESSURE, AND FORCE FOR TWO PARTICLES IN CONTACT}

The depletion effect leads to a monomer density profile that decreases on approaching the surface of a particle with ${ }^{10}$ the distance from the surface to the power $1 / \nu$. The prefactor of this power law is proportional to the local pressure which the polymer solution exerts onto the surface element considered. For two spherical particles $A, B$ the pressure $p$ acting on a surface element $d S_{A}$ of $A$ is determined by ${ }^{22}$

$$
B_{g} \frac{p}{k_{B} T}=\frac{\mathcal{R}_{g}^{1 / \nu}\langle\theta(\mathbf{r})\rangle_{A, B}^{(\mathrm{as})}}{\left(\left|\mathbf{r}-\mathbf{r}_{A}\right|-R\right)^{1 / \nu}}
$$

with the density $\theta$ from Eq. (1.2) and $B_{g}$ a universal densitypressure amplitude. ${ }^{15}$ Here \langle\rangle$_{A, B}$ denotes an average over chain configurations in the presence of the two particles and the superscript (as) means that $\mathbf{r}$ is-within the scaling regime-asymptotically close to the surface. The pressure $p=p(\cos \vartheta)$ depends on the projection $\cos \vartheta$ of the inward pointing surface normal onto the center-to-center distance vector $\mathbf{r}_{B}-\mathbf{r}_{A}$. Since this is the direction of the total force $\mathbf{f}$ pushing particle $A$ towards particle $B$ the magnitude $f$ of the force is

$$
\begin{aligned}
\partial F_{2} / \partial r_{A B}=f= & \int d S_{A} \cos \vartheta p \\
= & (2 R)^{d-1} \Omega_{d-1} \int_{0}^{\infty} d \tau\left(1-\tau^{2}\right) \\
& \times \tau^{d-2}\left(1+\tau^{2}\right)^{-d} p
\end{aligned}
$$

The explicit form of the integral over the surface $S_{A}$ as given for general dimension $d$ in the last expression of (3.2) involves

$$
\tau=\tan (\vartheta / 2)
$$

and the surface $\Omega_{d-1}=2 \pi^{(d-1) / 2} / \Gamma((d-1) / 2)$ of the sphere with radius 1 in $d-1$ dimensions.

The density-pressure relation (3.1) applies to situations with arbitrary ratios of mesoscopic lengths $R / \mathcal{R}_{g}, r_{A B} / \mathcal{R}_{g}$, and arbitrary overlap $s$. We now consider the limit $\mathcal{R}_{g} \rightarrow \infty$ with $R, r_{A B}$, and $s$ fixed in which the normalized monomer density $\mathcal{M}_{A, B}(\mathbf{r}) \equiv\langle\theta(\mathbf{r})\rangle_{A, B} / n$ and thus $p /\left(n \mathcal{R}_{g}^{1 / \nu}\right)$ and $f /\left(n \mathcal{R}_{g}^{1 / \nu}\right)$ become independent of $\mathcal{R}_{g}$ and $s$. This is consistent with the corresponding behavior (2.2)-(2.4) of $\delta F_{2}$. In this limit $\mathcal{M}_{A, B}$ can be obtained from the energy-density correlation function at the critical point of the corresponding field theory and by conformally mapping the outer space of the two particles onto simpler geometries. ${ }^{22}$ For two spherical particles in contact the outer space can be mapped onto the space between two parallel walls. For chains in two dimensions $d=2$ outside two circular disks and with monomer-monomer repulsion we now exactly calculate $\mathcal{M}_{A, B}$ from the known ${ }^{23}$ energy-density correlation function with the result

$$
\begin{aligned}
\mathcal{M}_{A, B}(\mathbf{r})= & \left(\frac{\pi^{2}}{4 \rho^{2} \cos \left(\pi \rho_{\|} / \rho^{2}\right)}\right)^{2 / 3} \frac{8 \pi}{45 \sqrt{3}} \frac{1}{\kappa} \\
& \times{ }_{3} F_{2}\left(\frac{1}{3}, \frac{2}{3}, \frac{7}{3} ; \frac{11}{6}, 2 ;-\frac{1}{4 \kappa}\right) .
\end{aligned}
$$

Here the contact point of the two disks with radius $R$ is the origin of the $\mathbf{r}$ coordinate system and $\rho_{\|} R$ and $\rho_{\perp} R$ are, respectively, the components of $\mathbf{r}$ parallel and perpendicular to the line connecting the contact point with the centers of the disks (so that the centers are located at $\rho_{\perp}=0$ with $\rho_{\|}$ $= \pm 1), \rho^{2}=\rho_{\|}^{2}+\rho_{\perp}^{2}$, and

$$
4 \kappa=\frac{\operatorname{Cosh}^{2}\left(\pi \rho_{\perp} / \rho^{2}\right)}{\cos ^{2}\left(\pi \rho_{\|} / \rho^{2}\right)}-1 .
$$

The hypergeometric function ${ }_{3} F_{2}$ has a convergent series representation for $4 \kappa>1$ and is defined for $4 \kappa<1$ by analytic continuation. ${ }^{24}$

Far from the two disks the density tends to its bulk value and the $\kappa \rightarrow 0$ behavior $^{24}$ in Eq. (3.4) implies a leading correction

$$
1-\mathcal{M}_{A, B} \rightarrow(\pi / 2)^{2 / 3} A_{g} \sigma_{g}(R / r)^{2 / 3}
$$

which is isotropic and is by a factor $m(d=2)=(\pi / 2)^{2 / 3}$ larger than for a single disk. The quantity $m$ is the same as in Eq. (2.6).

The upper and lower curves in Fig. 1 show the behavior of $\mathcal{M}_{A, B}$ in Eq. (3.4) along the lines $\rho_{\|}=0$ and $\rho_{\perp}=0$, respectively. On approaching the perimeter of disk $A$, $\cos \left(\pi \rho_{\|} / \rho^{2}\right)$ in Eqs. (3.4) and (3.5) approaches zero, $\kappa$ approaches infinity, and one finds from Eq. (3.1) with $\nu(d$ $=2)=3 / 4$ and from Eq. (3.3) the explicit expression 


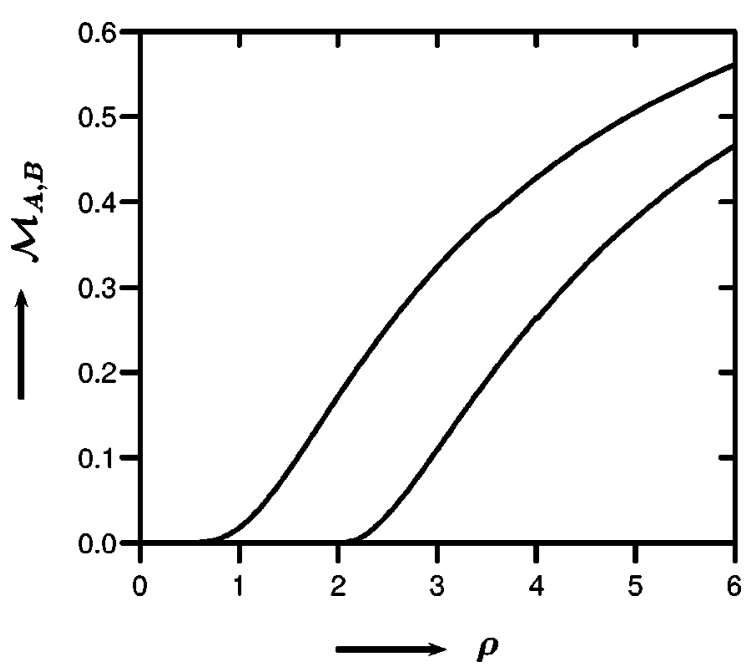

FIG. 1. Normalized monomer density $\mathcal{M}_{A, B}(\mathbf{r})$ for polymers in two dimensions outside two small circular disks in contact. The variation of $\mathcal{M}_{A, B}$ with distance $\rho R$ from the contact point is shown along the common tangent line of the two disks (upper curve) and along the line passing through the centers of the disks (lower curve). $\mathcal{M}_{A, B}$ approaches 1 for large $\rho$.

$$
B_{g} \frac{p}{k_{B} T}=n\left(\frac{\mathcal{R}_{g}}{R}\right)^{4 / 3}\left[\frac{1+\tau^{2}}{\operatorname{Cosh}(\tau \pi / 2)}\right]^{2} \frac{\pi^{11 / 3}}{45 \sqrt{3} 2^{1 / 3}}
$$

for the $\vartheta$-dependence of the local pressure. Near the perimeter point $\rho_{\perp}=0, \rho_{\|}=2$, where $\vartheta=0$ and the pressure is maximal, $p$ is proportional to $1-0.117 \vartheta^{2}$, while $p$ becomes exponentially small near the contact point where $\vartheta=\pi$. An integration over the perimeter $S_{A}$ as in Eq. (3.2) yields ${ }^{15}$

$$
\frac{f}{k_{B} T} /\left[n R\left(\frac{\mathcal{R}_{g}}{R}\right)^{4 / 3}\right]=\frac{1}{B_{g}} \frac{16 \pi^{8 / 3}}{135 \sqrt{3} 2^{1 / 3}} \approx 1.34
$$

for the magnitude $f$ of the total force on disk $A$. Approximating the force at contact by extrapolating Eqs. (2.2), (2.4) down to $r_{A, B}=2 R$ would lead in $d=2$ to an approximate value $A_{g}^{2} \sigma_{g} /\left(2^{2 / 3} 3\right)=1.98$ for the left-hand side of Eq. (3.8) which overestimates the correct value by about $50 \%$.

The results (3.7) and (3.8) for the polymer-induced pressure and force for two disks in contact should be compared with corresponding results for two spheres in four dimensions in which case one finds (apart from logarithmic corrections) the ideal chain behavior ${ }^{5}$ with $^{22}$

$$
B_{g} \frac{p}{k_{B} T}=n\left(\frac{\mathcal{R}_{g}}{R}\right)^{2} \frac{\pi^{4}}{2^{6}} \frac{\left(1+\tau^{2}\right)^{4}}{\tau^{2}} \frac{\operatorname{Sinh}^{2}(\tau \pi / 2)}{\operatorname{Cosh}^{4}(\tau \pi / 2)}
$$

and $^{15}$

$$
\frac{f}{k_{B} T} /\left[n R \mathcal{R}_{g}^{2}\right]=\pi^{2}\left(\frac{\pi^{2}}{6}-1\right)=6.36 .
$$

Here $p$ is proportional to $1-0.028 \vartheta^{2}$ near $\vartheta=0$. Note the different dimensions of $n$ in Eqs. (3.8) and (3.10).

In the case of ideal chains the amplitude of the force between small spheres vanishes as $d \rightarrow 2$. For two touching spheres in three dimensions we thus expect that the universal amplitude $f /\left(k_{B} \operatorname{Tn} R^{d-1-1 / \nu} \mathcal{R}_{g}^{1 / \nu}\right)$ for polymers in good solvent is larger than the value ${ }^{13}\left(\ln 2-\frac{1}{4}\right)(4 \pi / 3)=1.86$ of the corresponding amplitude $f /\left(k_{B} \operatorname{Tn} \mathcal{R}_{g}^{2}\right)$ for ideal $(\Theta-)$ poly- mers in $d=3$. A more quantitative estimate would follow from a $4-d$ expansion of the energy-density correlation function between parallel walls.

\section{SECOND VIRIAL COEFFICIENT}

Consider the contribution

$$
b_{2}^{\mathrm{CC}}=\frac{1}{2} \int_{\left(r_{A B}>2 R\right)} d \mathbf{r}_{A B}\left[1-\exp \left(-\frac{\delta F_{2}\left(r_{A B}\right)}{k_{B} T}\right)\right]
$$

to the second virial coefficient $B_{2}^{\mathrm{CC}}=B_{2}^{(\mathrm{HS})}+b_{2}^{\mathrm{CC}}$ of a dilute suspension of colloidal particles which arises from the polymer-induced potential of mean force $\delta F_{2}$. The hardsphere contribution $B_{2}^{(\mathrm{HS})}$ equals $16 \pi R^{3} / 3$ in three dimensions. For small particles we may expand the exponential in (4.1) and disregard the contribution from interparticle distances $r_{A B}$ of order $R$. From Eq. (2.2) we obtain

$$
2 b_{2}^{\mathrm{CC}} \rightarrow-\left(\frac{F_{1}}{k_{B} T n}\right)^{2} \int d \mathbf{r}_{A B} K\left(r_{A B}\right) .
$$

On using the compressibility sum rule ${ }^{6}$ for the density correlation function

$$
\int d \mathbf{r} K(r)=n /\left(\frac{\partial}{\partial n} \frac{\Pi}{k_{B} T}\right)
$$

and the scaling form

$$
\frac{\Pi}{k_{B} T}=n[1+\mathcal{P}(s)],
$$

where $\mathcal{P}$ is a universal scaling function for the osmotic pressure $\Pi$ of the monodisperse polymer solution without particles, one finds

$$
\frac{2 b_{2}^{\mathrm{CC}} \mathcal{R}_{g}^{d}}{\left(F_{1} /\left(k_{B} T n\right)\right)^{2}}=-\frac{s}{1+d[s \mathcal{P}(s)] / d s} \equiv \mathcal{B}(s) .
$$

Note that the $n$-dependence of the left-hand side of Eq. (4.5) comes exclusively from $b_{2}^{\mathrm{CC}}$. Equation (4.5) expresses $b_{2}^{\mathrm{CC}}$ in terms of the particle radius $R$, the amplitude $A_{g}$ in Eq. (1.1), and properties of the polymer solution without particles. An accurate expression for $\mathcal{B}$ in $d=3$ is available, see $\mathcal{P}(s)=\mathcal{P}^{*}(\hat{s},[m])$ and $d[s \mathcal{P}(s)] / d s=J_{c}^{*-1}(\hat{s},[m])-1$ in Eqs. (17.52) and (17.53) of Ref. 6 with $\hat{s}=3.584 s$. Figure 2 shows $\mathcal{B}(s)$ for three dimensions as a function of $n(4 \pi / 3) \mathcal{R}_{g}^{3}=(4 \pi / 3) s$, see Eq. (1.5). $\mathcal{B}(s)$ approaches $-s$ for small $s$, is proportional to $-s / s^{1 /(d \nu-1)}=-s^{-0.31}$ for large $s$, and displays a minimum at $(4 \pi / 3) s \approx 1$, where the chains "begin to overlap," and $\xi$ is of the order of $\mathcal{R}_{g}$. This implies a nonmonotonic $n$-dependence of $b_{2}^{\mathrm{CC}}$, qualitatively explained ${ }^{8}$ by Chatterjee and Schweizer as follows: On increasing the chain density $n$ within the dilute regime ( $s$ $\ll 1$ ), the coefficient $b_{2}^{\mathrm{CC}}$ drops, since the attractive potential of mean force $\delta F_{2}$ is proportional to $n$, see Eq. (2.2) with $K \propto n$ as mentioned below (2.5). After the chains begin to overlap, the range of $K$ is determined by $\xi$ and decreases on further increasing $n$ so that $b_{2}^{\mathrm{CC}}$ rises.

The value of the minimum of $\mathcal{B}$ is -0.049 and implies the relation 


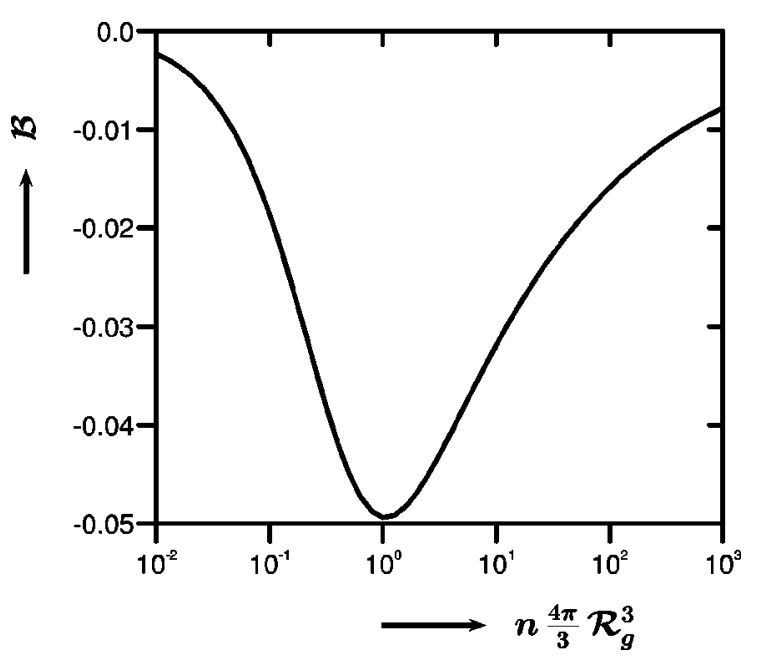

FIG. 2. Nonmonotonic overlap-dependence of the second virial coefficient $b_{2}^{\mathrm{CC}}$. Shown is the universal combination $\mathcal{B}$ in Eq. (4.5) for three dimensions as a function of $n(4 \pi / 3) \mathcal{R}_{g}^{3}$.

$$
\left(b_{2}^{\mathrm{CC}}\right)_{\min } / B_{2}^{(\mathrm{HS})}=-0.50\left(\mathcal{R}_{g} / R\right)^{0.401}
$$

for the coefficient $b_{2}^{\mathrm{CC}}$ at the polymer concentration where it is minimal. The exponent 0.401 is the value of $(2 / \nu)-d$ in three dimensions. This should be compared with the result $-0.066\left(\mathcal{R}_{g} / R\right)^{1}$ for the left-hand side of Eq. (4.6) which follows from Eq. (43) of Ref. 8 and is based on an integral equation approach. The exponents differ by more than a factor of 2 and the amplitudes by a factor of roughly 1/10. Thus the asymptotically exact result (4.6) for the minimum of $b_{2}^{\mathrm{CC}}$ of small colloidal particles $\left(R \ll \xi, \mathcal{R}_{g}\right)$ in a good solvent solution of long flexible chains close to their overlap concentration crosses the integral-equation based result at a value $\approx 29$ of $\mathcal{R}_{g} / R$ and leads to a minimum which is less deep (deeper) for $\mathcal{R}_{g} / R$ larger (smaller) than this value.

\section{INTERACTION BETWEEN A PARTICLE AND A WALL}

Experiments measuring the polymer-induced force on individual colloidal particles have been performed both for a single pair of interacting particles ${ }^{25}$ and for a single particle interacting with a wall. ${ }^{26,27}$ The free energy $F_{1, \mathrm{hs}}$ of immersion of a small particle in a polymer solution in a half space (hs) bounded by a planar wall can also be obtained by using Eq. (1.1). As in Sec. II, the free energy of a particle with its center at a distance $z_{C}$ from the wall

$$
F_{1, \mathrm{hs}}=F_{1} \mathcal{M}\left(z_{C}\right), \quad R \ll z_{C}, \mathcal{R}_{g}, \xi
$$

is determined by the bulk-normalized monomer density profile

$$
\mathcal{M}(z)=\langle\theta(\mathbf{r})\rangle_{\mathrm{hs}} / n
$$

in the solution in the half-space without the particle. Here \langle\rangle$_{\mathrm{hs}}$ denotes an average over all chain configurations in the half space. The quantity $\mathcal{M}(z)$ is independent of the component $\mathbf{r}_{\|}$parallel to the wall of the position vector $\mathbf{r}=\left(\mathbf{r}_{\|}, z\right)$ in Eq. (1.2) and approaches 1 for $z \rightarrow \infty$.
The dependence of $\mathcal{M}$ on $z, \mathcal{R}_{g}, \xi$ in the scaling regime is quite complex, in general, and we only consider special cases. Particularly transparent is the case of small $z$, in which $^{10,20}$

$$
k_{B} \operatorname{Tn} \mathcal{R}_{g}^{1 / \nu} \mathcal{M}(z) \rightarrow B_{g} \Pi z^{1 / \nu}, \quad z \ll \mathcal{R}_{g}, \xi
$$

with the bulk osmotic pressure $\Pi$ introduced in Eq. (4.4) and the universal density-pressure amplitude $B_{g}$ has the value ${ }^{15}$

$$
B_{g} \approx 1.07, \quad d=3 \text {, good solvent. }
$$

In the free energy formula (5.1) the relation (5.3) may only be used for the regime $R \ll z_{C} \ll \mathcal{R}_{g}, \xi$, while for a spherical particle touching the wall, i.e., for the smallest possible distance $z_{C}=R$,

$$
\left(F_{1 \mathrm{hs}}\right)_{z_{C}=R}=A_{\mathrm{ts}} \Pi R^{d}
$$

with a universal amplitude $A_{\mathrm{ts}}$ introduced in Ref. 20. The value of $A_{\text {ts }}$ for polymers in good solvent equals $\frac{1}{3} \pi^{3}=10.3$ in $d=2$ while in $d=4-\epsilon$ one finds

$$
\frac{A_{\mathrm{ts}}}{\left(A_{\mathrm{ts}}\right)_{\mathrm{id}}}=\frac{d-2}{d-1 / \nu}\left(1+\frac{\epsilon}{48}\right)
$$

to first order in $\epsilon$ with $\left(A_{\mathrm{ts}}\right)_{\mathrm{id}}=2 \pi^{d / 2}(d-1) \zeta(d) / \Gamma(d / 2)$ the value of $A_{\text {ts }}$ for ideal chains. Equation (5.6) follows from the known $^{28,29} \epsilon$-expansions of the quantities $\Delta$ and $C_{T}$ in Eq. (6.12) of Ref. 20 and suggests that $A_{\mathrm{ts}} \approx 23.7$ in $d=3$. Extrapolating Eqs. (5.1) and (5.3) down to $z_{C}=R$ leads to Eq.(5.5), with $A_{\text {ts }}$ replaced by the product $A_{g} B_{g}$. In $d=3$ this underestimates the amplitude in Eq. (5.5) by about $20 \%$.

The free energy (5.5) it costs to immerse the small particle close to the wall, i.e., in a region which is already depleted from polymers, is smaller by a factor $\propto\left(R / \mathcal{R}_{g}\right)^{1 / \nu} \Pi /\left(k_{B} T n\right)$ than the free energy (1.4) of immersion in the bulk of the polymer solution. The factor is proportional to $\left(R / \mathcal{R}_{g}\right)^{1 / \nu}$ and $(R / \xi)^{1 / \nu}$ for dilute and semidilute polymer solutions, respectively. This can be understood in terms of the effective pressure which the polymers exert on the surface of the particle. The pressure is proportional to the monomer density near the particle surface, ${ }^{10,22}$ compare Eq. (3.1), and determines the derivative of the free energy of immersion with respect to $R$. For the small particle in the bulk solution the pressure is ${ }^{22}$ proportional to $k_{B} \operatorname{Tn}\left(\mathcal{R}_{g} / R\right)^{1 / \nu}$ and is much larger than the pressure $\propto \Pi$ acting on a wall or a small particle close to a wall.

The variation of $F_{1 \text {,hs }}$ with particle wall distance $z_{C}$ has a point of inflection, since the upward curvature $\propto z_{C}^{1 / \nu}=z_{C}^{1.70}$ at small distances is followed by a downward curvature at large $z_{C}$ so that $F_{1, \text { hs }}$ approaches the $z_{C}$-independent bulk free energy $F_{1}$ for $z_{C} \rightarrow \infty$. Thus the magnitude $\partial F_{1, \mathrm{hs}} / \partial z_{C}$ of the polymer mediated mean force between wall and particle has a maximum at a distance of order $\mathcal{R}_{g}$ or $\xi$ in a dilute or semidilute polymer solution.

\section{SUMMARY AND CONCLUDING REMARKS}

We have discussed the polymer-induced depletion interactions between two particles and between a particle and a wall for dilute and semidilute solutions of long flexible polymer chains in a good solvent and mesoscopic spherical par- 
ticles with a small radius $R$. In particular we consider the attractive free energy of interaction (potential of mean force) $\delta F_{2}$ between two particles, the resulting contribution $b_{2}^{\mathrm{CC}}$ to the second virial coefficient of a dilute suspension of particles, and the free energy $F_{1 \text {,hs }}$ it costs to immerse a particle at a distance $z_{C}$ from a planar boundary wall of a polymer solution in the half-space. For two spherical particles in contact the depletion of the monomer density and the variation of the local pressure along the particle surfaces have also been investigated. Results for these universal quantities are presented which contain no free parameters or unknown prefactors and apply for arbitrary interchain overlap ranging from zero (dilute solution) to infinity (semidilute limit).

Using small radius expansions (which are similar to "short distance expansions" in field theories) we have shown that the overlap dependence of both $b_{2}^{\mathrm{CC}}$ and $F_{1 \text {,hs }}$ for a particle close to a wall can be expressed in terms of the overlap dependence of the osmotic pressure $\Pi$ of the polymer solution without particles and walls. The dependence of $b_{2}^{\mathrm{CC}}$ is determined by the compressibility of the polymer solution, see Eq. (4.5) and Fig. 2. The minimum value of the reduced virial coefficient $b_{2}^{\mathrm{CC}} / R^{3}$ at the polymer overlapconcentration depends on $\mathcal{R}_{g} / R$, with $\mathcal{R}_{g}$ the radius of gyration of one chain in dilute solution, as a power law, with an exponent and amplitude quite different from the predictions of an integral-equation approach, ${ }^{8}$ see Eq. (4.6). For $\mathcal{R}_{g} / R$ larger (smaller) than $\approx 29$ the minimum is less deep (deeper) than the minimum in the integral-equation approach.

We have also calculated the free energy of interaction and the force for two particles (and for a particle and a wall) in contact. In the case of three dimensions $d=3$ we estimate the free energy amplitudes from $\epsilon$-expansions, compare Eqs. (2.7) and (5.6), and conjecture an inequality for the force between two particles, compare the last paragraph in Sec. III. In the case of two dimensions $d=2$ and for two particles (disks) we derive exact results for the monomer-density depletion profile, the surface pressure, and the force between the particles; compare Eqs. (3.4)-(3.8) and Fig. 1. These results for contact-situations cannot be inferred from smallradius expressions such as Eqs. (2.2), (2.4) or (5.1), (5.3) which are obtained via Eq. (1.1) and are valid for particlecenter to particle-center distance $r_{A, B}$ (or particle-center to wall distance $z_{C}$ ) much smaller than $\mathcal{R}_{g}, \xi$, but much larger than the particle radius $R$. Extrapolating these expressions down to $r_{A, B}=2 R$ (or to $z_{C}=R$ ) in general overestimates the magnitude of the free energy of interaction and the force of two particles in contact. It also underestimates both the free energy to insert a particle in contact with a wall and the force on this particle. Compare the force between two disks in contact which is overestimated by about $50 \%$ as discussed below Eq. (3.8) and the free energy $F_{1, \text { hs }}$ it costs to insert a sphere in contact with a wall in three dimensions which is underestimated by about $20 \%$ as discussed below Eq. (5.6).

Equations such as (2.2), (4.2), and (5.1), which relate properties of small particles in the polymer solution to properties of the solution without particles, are expected to apply even beyond the asymptotic good solvent scaling region (excluded-volume limit). In a simplified description of the crossover from theta to excluded volume behavior in which chains at the theta point in $d=3$ are modeled as ideal, random walk chains, the relative reduction $1-W_{C}$ of the Boltzmann weight of chain configurations due to the presence of the particle in Eq. (1.1) can again be written as a product of the configuration-dependent chain number density $\theta$ in Eq. (1.2) and a configuration-independent factor depending on the particle radius $R$. This factor now also depends on the strength of the excluded volume interaction between chain monomers, ${ }^{30}$ and for weak interaction, corresponding to a solution close to the theta point with a large crossover length $\lambda$, crosses over from ideal chain behavior $A_{g}^{(\mathrm{id})} R^{d-2} \mathcal{R}_{g \text {, id }}^{2}$ for $R \ll \lambda$ to the excluded volume behavior $A_{g} R^{d-1 / \nu} \mathcal{R}_{g}^{1 / \nu}$ of Eq. (1.1) for $R \gg \lambda$. This complicated factor drops out of the ratios $\delta F_{2} / F_{1}^{2}, b_{2}^{\mathrm{CC}} / F_{1}^{2}$, and $F_{1, \text { hs }} / F_{1}$, which for arbitrary $R / \lambda$ are completely determined by crossover-properties of the polymer solution without particles according to Eqs. (2.2), (4.2), and (5.1).

It would be interesting to compare these new predictions with computer simulations ${ }^{31}$ in the appropriate region of mesoscopic length ratios and with real experiments. $3,4,25-27$

\section{ACKNOWLEDGMENTS}

It is a pleasure to thank A. Bringer and T. Burkhardt for useful discussions.

${ }^{1}$ R. Tuinier et al., Phys. Rev. E 60, 848 (1999).

${ }^{2}$ J. Janzen and D. E. Brooks, Clin. Hemorheol. 9, 695 (1989).

${ }^{3}$ A. M. Kulkarni, A. P. Chatterjee, K. S. Schweizer, and C. F. Zukoski, Phys. Rev. Lett. 83, 4554 (1999); J. Phys. (London) C 12, A301 (2000).

${ }^{4}$ While on sufficiently large length scales a model with only short range forces applies even in the presence of dispersion forces, in practice it is not easy to reach this "asymptotic region."

${ }^{5}$ P. G. de Gennes, Scaling Concepts in Polymer Physics (Cornell University, Ithaca, 1979).

${ }^{6}$ L. Schaefer, Excluded Volume Effects in Polymer Solutions (Springer, Berlin, 1999).

${ }^{7}$ A. Bringer, E. Eisenriegler, F. Schlesener, and A. Hanke, Eur. Phys. J. B 11, 101 (1999).

${ }^{8}$ A. P. Chatterjee and K. S. Schweizer, J. Chem. Phys. 109, 10464 (1998).

${ }^{9}$ S. Asakura and F. Oosawa, J. Chem. Phys. 22, 155 (1954); J. Polym. Sci. 33, 183 (1958).

${ }^{10}$ J. F. Joanny, L. Leibler, and P. G. de Gennes, J. Polym. Sci., Polym. Phys. Ed. 17, 1073 (1979).

${ }^{11}$ A. McPherson, Methods Enzymol. 114, 112 (1985).

${ }^{12}$ E. Eisenriegler, A. Hanke, and S. Dietrich, Phys. Rev. E 54, 1134 (1996).

${ }^{13}$ A. Hanke, E. Eisenriegler, and S. Dietrich, Phys. Rev. E 59, 6853 (1999).

${ }^{14}$ One way to estimate the excluded volume effects between chainmonomers in good solvent in $d=3$ is by interpolating between $d=4$ where they can be neglected (Refs. 5 and 6) and $d=2$ where a number of exact results is available.

${ }^{15}$ The amplitudes $A_{g}=\left(\mathcal{R}_{x} / \mathcal{R}_{g}\right)^{1 / \nu} A_{D, D}, \quad \sigma_{g}=\left(\mathcal{R}_{g} / \mathcal{R}_{x}\right)^{1 / \nu} \sigma$, and $B_{g}$ $=\left(\mathcal{R}_{g} / \mathcal{R}_{x}\right)^{1 / \nu} B$ are related to the amplitudes $A_{D, D}, \sigma$, and $B$ considered in Table I of Ref. 13 and in the paragraph containing Eq. (5.12) in Ref. 20 via the ratio $\mathcal{R}_{x}^{2} / \mathcal{R}_{g}^{2}=\left(\mathcal{R}_{e e}^{2} / d\right) / \mathcal{R}_{g}^{2}$, where $\mathcal{R}_{e e}^{2}$ is the mean square endto-end distance. For good solvent conditions in three dimensions the amplitudes in Eqs. (1.3), (2.5), and (3.4) follow from $A_{3,3} \approx 9.82, \sigma \approx 0.13$, $B \approx 2$, and (Ref. 6) $\mathcal{R}_{x}^{2} / \mathcal{R}_{g}^{2} \approx 2.09, \nu \approx 0.588$ implying $\left(\mathcal{R}_{x} / \mathcal{R}_{g}\right)^{1 / \nu}$ $\approx 1.87$. In two dimensions $\left(\mathcal{R}_{x} / \mathcal{R}_{g}\right)^{1 / \nu} \approx 2.34$ [see J. Cardy and A. Guttmann, J. Phys. (London) A 26, 2485 (1993)] and the amplitudes follow from $A_{2,2} \approx 3.810, \quad \sigma \approx 0.278$, and $B \approx 2.01$. In four dimensions $\left(\mathcal{R}_{x} / \mathcal{R}_{g}\right)^{1 / \nu}=3 / 2, A_{4,4}=19.74$, and $B=2$.

${ }^{16}$ P. G. de Gennes, C.R. Acad. Sci. Paris B 288, 359 (1979).

${ }^{17}$ T. Odijk, Macromolecules 29, 1842 (1996); J. Chem. Phys. 106, 3402 (1996); Physica A 278, 347 (2000).

${ }^{18}$ A. George and W. W. Wilson, Acta Crystallogr., Sect. D: Biol. Crystallogr. 50, 361 (1994). 
${ }^{19}$ R. P. Sear, Eur. Phys. J. B 1, 313 (1998).

${ }^{20}$ E. Eisenriegler, in Field Theoretical Tools in Polymer- and ParticlePhysics, Lecture Notes in Physics, edited by H. Meyer-Ortmanns and A. Klümper (Springer, Berlin, 1998), Vol. 508.

${ }^{21}$ M. Krech, E. Eisenriegler, and S. Dietrich, Phys. Rev. E 52, 1345 (1995).

${ }^{22}$ E. Eisenriegler, J. Phys. (London) C 12, A227 (2000).

${ }^{23}$ T. Burkhardt, E. Eisenriegler, and I. Guim, Nucl. Phys. B 316, 559 (1989); T. Burkhardt and E. Eisenriegler, ibid. 424, 487 (1994).

${ }^{24}$ The two leading contributions for $\kappa \rightarrow 0$ of $(8 \pi / 45 \sqrt{3}) \kappa^{-1 / 3}{ }_{3} F_{2}\left(\frac{1}{3}, \frac{2}{3}, \frac{7}{3} ; \frac{11}{6}, 2 ;-(1 / 4 \kappa)\right)$ are given by $1-\left|B_{2}\right| \kappa^{1 / 3}$ with a short distance amplitude (Ref. 23), $\left|B_{2}\right|=4 \sqrt{\pi}$ $\left[\Gamma\left(\frac{2}{3}\right) / \Gamma\left(\frac{1}{6}\right)\right]^{2}|\Gamma(-1 / 6)| / \Gamma\left(\frac{1}{3}\right) \approx 1.05$ which equals (Ref. 13) the product
$A_{g} \sigma_{g}$ of the amplitudes in Eqs. (1.1) and (2.4) for $d=2$.

${ }^{25}$ R. Verma, J. C. Crocker, T. C. Lubensky, and A. G. Yodh, Phys. Rev. Lett. 81, 4004 (1998).

${ }^{26}$ Y. N. Ohshima et al., Phys. Rev. Lett. 78, 3963 (1997).

${ }^{27}$ D. Rudhardt, C. Bechinger, and P. Leiderer, Phys. Rev. Lett. 81, 1330 (1998)

${ }^{28}$ M. Krech and S. Dietrich, Phys. Rev. A 46, 1886 (1992).

${ }^{29}$ E. Eisenriegler, M. Krech, and S. Dietrich, Phys. Rev. B 53, 14377 (1996).

${ }^{30}$ The product form of $1-W_{C}$ was checked in a one-loop calculation in Appendix B of Ref. 13 making use of the polymer-magnet analogy (Refs. 5 and 20). The factor multiplying $\theta$ in $1-W_{C}$ is given by $\mathcal{F}(\mu R, u, d) \mu^{2-d} Z_{t} 6 \mathcal{R}_{g, \text { id }}^{2} / d$ in the notation of Ref. 13.

${ }^{31}$ R. Dickman and A. Yethiraj, J. Chem. Phys. 100, 4683 (1994). 